\title{
Entanglement of a Laguerre-Gaussian cavity mode with a rotating mirror
}

\author{
M. Bhattacharya, P.-L. Giscard, and P. Meystre \\ B2 Institute, Department of Physics and College of Optical Sciences, \\ The University of Arizona, Tucson, Arizona 85721
}

(Dated: November 27, 2018)

\begin{abstract}
It has previously been shown theoretically that the exchange of linear momentum between the light field in an optical cavity and a vibrating end mirror can entangle the electromagnetic field with the vibrational motion of that mirror. In this paper we consider the rotational analog of this situation and show that radiation torque can similarly entangle a Laguerre-Gaussian cavity mode with a rotating end mirror. We examine the mirror-field entanglement as a function of ambient temperature, radiation detuning and orbital angular momentum carried by the cavity mode.
\end{abstract}

PACS numbers: $03.65 . \mathrm{Ud}, 42.50 . \mathrm{Pq}, 42.50 . \mathrm{Lc}, 45.20 . \mathrm{dc}$ 


\section{INTRODUCTION}

The optomechanical driving of optical cavities with laser radiation is an experimentally promising method for eliciting quantum mechanical behavior from classical objects [1, 2, 3, 4, 5]. In the case of a two-mirror cavity with a perfectly reflecting end-mirror mounted on a cantilever and allowed to vibrate along the cavity axis, laser radiation entering through the slightly transmissive fixed input mirror can both trap and cool the mirror motion, depending on whether it is detuned slightly above or below a cavity resonance [5]. Radiation pressure can thus decrease the number of phonons in the vibrating mirror,

$$
n_{m}=\frac{k_{B} T_{\text {eff }}}{\hbar \omega_{\text {eff }}}
$$

since cooling lowers the effective temperature of the mirror $T_{\text {eff }}$ while trapping increases its effective vibration frequency $\omega_{\text {eff }}$. A key goal is to achieve $n_{m}<1$, i.e. to place the mirror near its quantum mechanical ground state. Evidence of reaching that regime could come from measuring $n_{m}$, and a strategy for doing so has recently been proposed [6].

Radiation pressure can play an additional important role as a mechanism for generating entanglement. In addition to its basic interest, quantum entanglement - the existence of correlations disallowed by classical physics between two objects - has been demonstrated to be a valuable resource for quantum information processing tasks such as teleportation and superdense coding (see Ref. [7] and references therein).

It has been shown theoretically that radiation pressure can entangle a light field to a

vibrating mirror both in the absence [8] and in the presence 9] of a cavity. It can also entangle two vibrating mirrors, both when they form a cavity [10, 11] and when they do not [12, 13]. A single vibrating mirror can also entangle two [14, 15] or more [16] light fields. The teleportation of quantum states from a light field to a vibrating mirror has been proposed in Ref. [17].

In these proposals the physical mechanism behind the generation of entanglement is the exchange of linear momentum between the photons in the cavity and the mirror. In recent work, we have proposed a rotational analog of mirror cooling and trapping where the light-mirror interaction relies on the exchange of angular momentum between a LaguerreGaussian cavity mode and a spiral phase element used as a cavity end-mirror [18]. This article further investigates that system and shows how the radiation torque can generate 


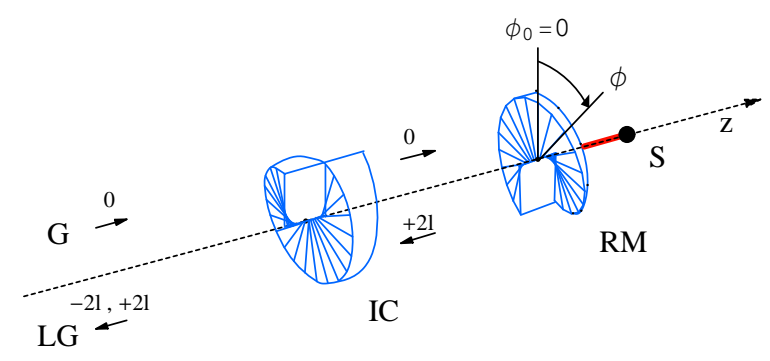

FIG. 1: (Color online). Arrangement for entangling the rotation of a mirror with a LaguerreGaussian (LG) mode using angular momentum exchange. A Gaussian (G) field of optical charge 0 enters the cavity through a fixed partially transparent input coupler (IC) that does not affect its charge. A perfectly reflecting rear mirror $(\mathrm{RM})$ rotating about the cavity axis on a support $\mathrm{S}$ adds a charge $2 l$ to the beam on reflection. Both the IC and the RM are spiral phase elements. The angular deflection of the RM from equilibrium $\left(\phi_{0}=0\right)$ is indicated by the angle $\phi$. The charge on the beams at various points is also indicated.

entanglement between the optically charged light field and a rotating mirror. We investigate the entanglement as a function of ambient temperature, detuning of the laser radiation, and orbital angular momentum carried by the cavity mode.

The rest of the article is organized as follows. Section [I] briefly reviews the cavity configuration proposed in Ref. [18]. Section [II discusses the steady state of the system, discusses the linear fluctuations of the field and mirror motion about that steady state, and examines the dynamical stability of the rotating mirror. Section IV demonstrates the onset of entanglement between the intra-cavity Laguerre-Gaussian mode and the rotating end-mirror and discusses its dependence on ambient temperature, detuning, and angular momentum of the light field. We also briefly discuss the experimental measurement of the entanglement. Section $\mathrm{V}$ provides a summary and a conclusion. Appendix $\mathrm{A}$ derives and solves the Lyapunov equation for the correlation matrix determining the entanglement between the mirror and the cavity mode.

\section{CAVITY MODEL}

The optomechanical system under consideration is described in detail in Ref. [18] and illustrated in Fig. 1, Both cavity mirrors are spiral phase elements [19]. The input mirror 
is slightly transmissive but does not change the orbital angular momentum of any beam passing through it. In reflection, however, it removes a charge $2 l$ from the beam. Likewise the end mirror, which is assumed to be perfectly reflecting, is designed to add a charge $2 l$ to a beam on reflection. With these specifications it can be seen that a Gaussian beam (with no charge) incident on the cavity can transfer a torque $\xi_{\phi}=c l \hbar / L$ per photon to the end mirror, where $c$ is the velocity of light and $L$ is the length of the cavity [18].

In dimensionless units, the Hamiltonian that describes this system is [18]

$$
H=\hbar \omega_{c} a^{\dagger} a+\frac{\hbar \omega_{\phi}}{2}\left(L_{z}^{2}+\phi^{2}\right)-\hbar g a^{\dagger} a \phi
$$

where $a$ and $a^{\dagger}$ are the bosonic annihilation and creation operators for the cavity mode of frequency $\omega_{c}, L_{z}$ and $\phi$ are the angular momentum and angular displacement, respectively, of the rear mirror about the cavity axis, with $\left[L_{z}, \phi\right]=-i$. Finally $\omega_{\phi}$ is the angular rotation frequency of the rear mirror and

$$
g=\frac{c l}{L} \sqrt{\frac{\hbar}{I \omega_{\phi}}}
$$

is the optorotational coupling parameter, with $I=M R^{2} / 2$ the moment of inertia of the mirror of mass $M$ and radius $R$ about the cavity axis.

\section{QUANTUM DYNAMICS}

The evolution of the operators describing the dynamics of the intracavity field and the mirror motion is given by quantum Langevin equations accounting for noise and damping due to the vacuum fluctuations entering the cavity field as well as to the Brownian noise coupling to the moving mirror [18],

$$
\begin{aligned}
\dot{a} & =-i(\delta-g \phi) a-\frac{\gamma}{2} a+\sqrt{\gamma} a^{\mathrm{in}}, \\
\dot{\phi} & =\omega_{\phi} L_{z}, \\
\dot{L}_{z} & =-\omega_{\phi} \phi+g a^{\dagger} a-\frac{D_{\phi}}{I} L_{z}+\epsilon^{\mathrm{in}} .
\end{aligned}
$$

where $\delta=\omega_{c}-\omega_{L}$ is the detuning of the laser frequency $\omega_{L}$ from the cavity resonance, $\gamma$ is the cavity damping rate and $D_{\phi}$ is the intrinsic damping constant of the rotating mirror. The noise operator $a^{\text {in }}$ describes the laser field incident on the cavity, of mean amplitude $\left\langle a^{\text {in }}(t)\right\rangle=a_{\mathrm{s}}^{\text {in }}$. Its fluctuations are taken to be delta-correlated,

$$
\left\langle\delta a^{\text {in }}(t) \delta a^{\text {in }, \dagger}\left(t^{\prime}\right)\right\rangle=\delta\left(t-t^{\prime}\right)
$$


and add vacuum noise to the cavity modes. The Brownian noise operator $\epsilon^{\text {in }}$ describes the mechanical noise that couples to the mirror from the environment. Its mean value is zero and its fluctuations are correlated at temperature $T$ as [20]

$$
\begin{aligned}
& \left\langle\delta \epsilon^{\mathrm{in}}(t) \delta \epsilon^{\mathrm{in}}\left(t^{\prime}\right)\right\rangle= \\
& \frac{D_{\phi}}{\omega_{\phi} I} \int_{-\infty}^{\infty} \frac{d \omega}{2 \pi} e^{-i \omega\left(t-t^{\prime}\right)} \omega\left[1+\operatorname{coth}\left(\frac{\hbar \omega}{2 k_{\mathrm{B}} T}\right)\right],
\end{aligned}
$$

where $k_{\mathrm{B}}$ is Boltzmann's constant. For high mechanical quality, $\left(\omega_{\phi} I / D_{\phi} \gg 1\right)$, the fluctuations become delta-correlated and Eq. (5) simplifies to

$$
\left\langle\delta \epsilon^{\mathrm{in}}(t) \delta \epsilon^{\mathrm{in}}\left(t^{\prime}\right)\right\rangle=\frac{D_{\phi}}{I}(2 \bar{n}+1) \delta\left(t-t^{\prime}\right)
$$

where

$$
\bar{n}=\left(e^{\hbar \omega_{\mathrm{eff}} / k_{B} T}-1\right)^{-1}
$$

is the mean number of thermal phonons available at the mirror of effective rotation frequency $\omega_{\text {eff }}$ and temperature $T$ [see Eq. (29) for an expresion for $\omega_{\text {eff }}$. The thermal phonon spectrum peaks approximately at the temperature

$$
T_{c} \sim \frac{\hbar \omega_{\mathrm{eff}}}{4 k_{B}}
$$

We show later on that it is possible to maintain a mirror-field entanglement close to its maximum value for $T<T_{c}$, but that that entanglement decreases sharply beyond $T \sim T_{c}$ (Fig. 3). This can be understood intuitively by noting that for $T>T_{c}$ we obtain $\omega>\omega_{\text {eff }}$ and the mirror can be rotationally excited by thermal phonons, but this ceases to be the case for $T<T_{c}$, in which case $\omega<\omega_{\text {eff }}$.

\section{A. Steady state}

The semiclassical, steady-state solution of Eq.(4) is

$$
\begin{aligned}
a_{s} & =\frac{\sqrt{\gamma} a_{s}^{\text {in }}\left[\gamma / 2-i\left(\delta-g \phi_{s}\right)\right]}{(\gamma / 2)^{2}+\left(\delta-g \phi_{s}\right)^{2}} \\
\phi_{s} & =\frac{g\left|a_{s}\right|^{2}}{\omega_{\phi}}, \\
L_{z, s} & =0 .
\end{aligned}
$$


The steady state field amplitude $a_{s}$ is complex in general, but in simple situations where the incident field is kept constant throughout, it is possible to chose its phase such that $a_{s}$ is real,

$$
a_{\mathrm{s}}=\frac{\sqrt{\gamma}\left|a_{\mathrm{s}}^{\mathrm{in}}\right|}{\left[\left(\frac{\gamma}{2}\right)^{2}+\left(\delta-g \phi_{\mathrm{s}}\right)^{2}\right]^{1 / 2}} .
$$

We restrict our discussion to that situation here. The steady-state is bistable for high enough incident field [21, 22], but we assume in the following that an electronic feedback loop maintains the length of the cavity and hence removes bistability. This is a standard practice in current experiments on optomechanical cooling [1, 2, 표, 4].

\section{B. Fluctuations}

To account for the quantum fluctuations about the semiclassical steady-state we expand the Heisenberg operator $a$ about the $a_{s}$ state in the usual way as $a=a_{s}+\delta a$, and similarly for the other operators. Linearizing the quantum Langevin equations of motion in the fluctuations gives then

$$
\begin{aligned}
\delta \dot{X} & =-\frac{\gamma}{2} \delta X+\Delta \delta Y+\sqrt{\gamma} \delta X_{a}^{\mathrm{in}} \\
\delta \dot{Y} & =-\frac{\gamma}{2} \delta Y-\Delta \delta X+G \delta \phi+\sqrt{\gamma} \delta Y_{a}^{\mathrm{in}} \\
\dot{\delta \phi} & =\omega_{\phi} \delta L_{z}, \\
\delta \dot{L}_{z} & =-\omega_{\phi} \delta \phi+G \delta X-\frac{D_{\phi}}{I} \delta L_{z}+\delta \epsilon^{\mathrm{in}},
\end{aligned}
$$

where $\Delta=\delta-g \phi_{s}$ is the effective cavity detuning, $G=g a_{s} \sqrt{2}$ is the effective optorotational parameter, and we have redefined the field fluctuations in terms of their quadratures as

$$
\begin{aligned}
& \delta X_{a}=\left(\delta a+\delta a^{\dagger}\right) / \sqrt{2}, \\
& \delta Y_{a}=\left(\delta a-\delta a^{\dagger}\right) / i \sqrt{2}
\end{aligned}
$$

Eq. (11) can be recast compactly as

$$
\dot{u}(t)=B u(t)+n(t),
$$


where $u(t)=\left(\delta \phi, \delta L_{z}, \delta X_{a}, \delta Y_{a}\right)$ is the vector of fluctuations, $n(t)=$ $\left(0, \delta \epsilon^{\mathrm{in}}, \sqrt{\gamma} \delta X_{a}^{\mathrm{in}}, \sqrt{\gamma} \delta Y_{a}^{\mathrm{in}}\right)$ is the input noise vector, and

$$
B=\left(\begin{array}{cccc}
0 & \omega_{\phi} & 0 & 0 \\
-\omega_{\phi} & -D_{\phi} / I & G & 0 \\
0 & 0 & -\gamma / 2 & \Delta \\
G & 0 & -\Delta & -\gamma / 2
\end{array}\right) .
$$

This matrix determines the dynamic stability of the physical system, and also a measure of the entanglement between its two subsystems, the intracavity field and the rotating mirror.

\section{Dynamic stability}

According to the Routh-Hurwitz criterion, the stability of the steady-state solution is assured if none of the eigenvalues of the matrix $B$ has a positive real part. This requirement can be reframed as a series of inequalities that have to be obeyed by the matrix elements of $B$ [23]. These inequalities, given by

$$
\begin{aligned}
& D_{\phi} I^{2} \gamma\left[16 \omega_{\phi}^{4}+32 G^{2} \omega_{\phi} \Delta+8 \omega_{\phi}^{2}\left(\gamma^{2}-4 \Delta^{2}\right)\right. \\
+ & \left.\left.\left(\gamma^{2}+4 \Delta^{2}\right)^{2}\right)\right]+16 G^{2} I^{3} \omega_{\phi} \gamma^{2} \Delta+4 D_{\phi}^{3}\left(\gamma^{3}+4 \gamma \Delta^{2}\right) \\
+ & 4 D_{\phi}^{2} I\left(4 \omega_{\phi}^{2} \gamma^{2}+\gamma^{4}+4 G^{2} \omega_{\phi} \Delta+4 \gamma^{2} \Delta^{2}\right)>0, \\
& \omega_{\phi}\left(\gamma^{2}+4 \Delta^{2}\right)-4 G^{2} \Delta>0 .
\end{aligned}
$$

have been numerically verified to hold for the parameters of this article.

\section{ENTANGLEMENT}

We quantify the entanglement between the cavity mode and the rotating end mirror in terms of the logarithmic negativity $E_{N}$, a measure of entanglement that was proposed in Ref. [24] to describe continuous Gaussian variables and has been evaluated in a number

of examples [9, 25, 26, 27]. The logarithmic negativity can be expressed in terms of the elements of the correlation matrix

$$
C_{i j}=\left[\left\langle u_{i}(\infty) u_{j}(\infty)+u_{j}(\infty) u_{i}(\infty)\right\rangle\right] / 2 .
$$


For the problem at hand, $C$ is a $4 \times 4$ matrix that can be cast in the form

$$
C=\left(\begin{array}{cc}
R & F \\
F^{T} & S
\end{array}\right)
$$

where $R, F$ and $S$ are $2 \times 2$ matrices. In terms of these matrices the logarithmic negativity is $[24]$

$$
E_{N}=\max \left[0,-\ln \left(2 \eta^{-}\right)\right]
$$

where

$$
\eta^{-}=2^{-1 / 2}\left(\sigma-\left[\sigma^{2}-4|C|\right]^{1 / 2}\right)^{1 / 2}
$$

and $\sigma=|R|+|S|-2|F|,|R|$ being the determinant of the matrix $R$. Quantum entanglement occurs for $E_{N}>0$, i.e. for $\eta^{-}<1 / 2$.

The bipartite entanglement between the cavity mode and the rotating mirror can be evaluated directly from the knowledge of the matrix $B$ [9]. Integrating Eq. (12) formally we have

$$
u(t)=M(t) u(0)+\int_{0}^{t} d s M(s) n(t-s),
$$

where $M(s)=\exp (B s)$. When the inequalities in Eq.(14) are obeyed the system is stable, as we have seen, hence $M(\infty)=0$ so that

$$
u(\infty)=\lim _{t \rightarrow \infty} \int_{0}^{t} d s M(s) n(t-s),
$$

and the steady state is independent of the initial conditions $u(0)$ as it should be.

The stationary quantum fluctuations are zero-mean Gaussian processes fully characterized by the $4 \times 4$ correlation matrix $C_{i j}$ which reads, with Eq. (20),

$$
C_{i j}=\sum_{k, l} \int_{0}^{\infty} d s \int_{0}^{\infty} d s^{\prime} M_{i k}(s) M_{j l}\left(s^{\prime}\right) \Phi_{k l}\left(s-s^{\prime}\right),
$$

where

$$
\Phi_{k l}\left(s-s^{\prime}\right)=\left(\left\langle n_{k}(s) n_{l}\left(s^{\prime}\right)+n_{l}(s) n_{k}\left(s^{\prime}\right)\right\rangle\right) / 2
$$

characterizes the stationary noise correlations. In the limit of a large mechanical quality factor, Eq. (6) yields readily $\Phi_{k l}\left(s-s^{\prime}\right)=D_{k l} \delta\left(s-s^{\prime}\right)$, where

$$
D=\left(\begin{array}{cccc}
0 & 0 & 0 & 0 \\
0 & D_{\phi}(2 \bar{n}+1) / I & 0 & 0 \\
0 & 0 & \gamma / 2 & 0 \\
0 & 0 & 0 & \gamma / 2
\end{array}\right)
$$




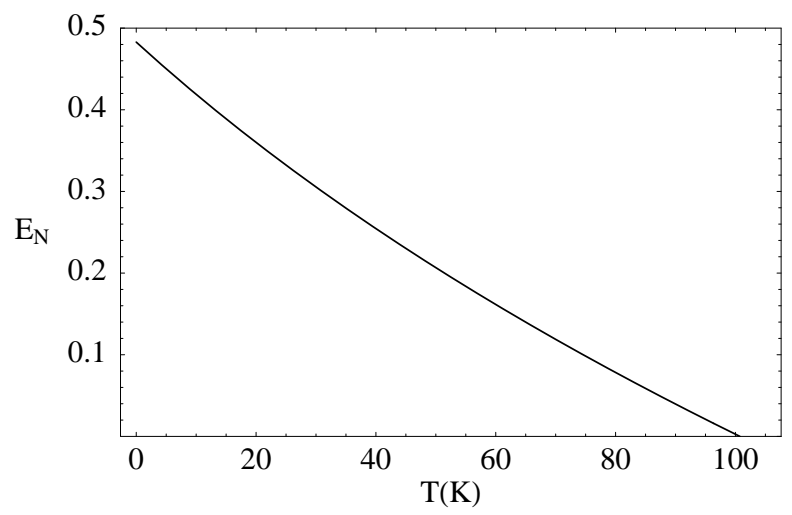

FIG. 2: Logarithmic negativity $E_{N}$ as a function of ambient temperature, for the parameters of Table I.

and Eq. (21) simplifies to

$$
C=\int_{0}^{\infty} d s M(s) D M(s)^{T}
$$

which can be integrated by parts to give

$$
B C+C B^{T}=-D
$$

see Appendix A. Using Eqs. (13) and (22), Eq. (24) can be solved analytically to yield the correlation matrix $C$, see Appendix $\mathrm{A}$. The full solution is quite complicated and its form is not particularly instructive, hence it will not be reproduced here.

We have evaluated numerically the logarithmic negativity $E_{N}$ for various parameters. Our main results are summarized in Figs. 2 4 for the parameter values of Table. I, where the mechanical quality is

$$
Q_{\phi}=\frac{\omega_{\phi}}{D_{\phi} / I}
$$

and $F$ is the optical finesse. Our parameters are somewhat similar to the case of the linearly vibrating mirror of Ref. [9], hence we make comparisons between specific figures in that article and the present one when appropriate.

\section{A. High temperature regime}

Fig. 2 shows $E_{N}$ as a function of the temperature $T$ of the mirror environment. Our results, which resemble those reported in Fig. 2 of Ref. [9], indicate that quantum entanglement persists until roughly $100 \mathrm{~K}$, that is, it should therefore be measurable in experiments in cryogenic environments. 


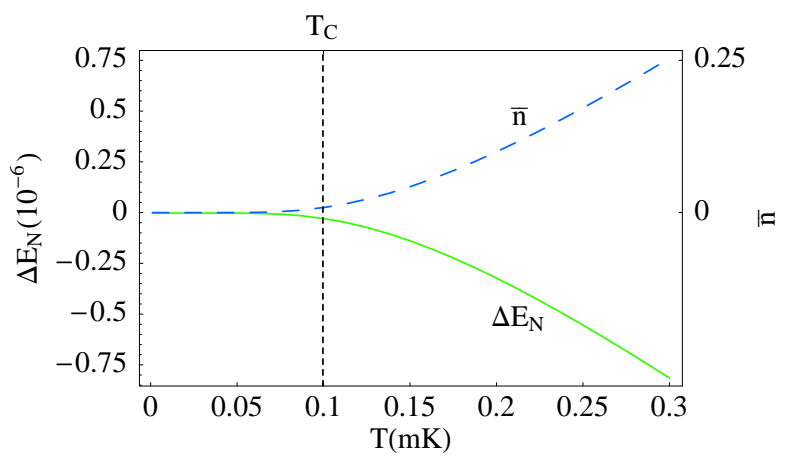

FIG. 3: (Color online) Solid line: Deviation $\Delta E_{N}$ of $E_{N}$ from its maximum value $E_{0} \sim 0.5$ as a function of $T$. The graph shows the low temperature end of the curve. Dashed line: mean number of thermal phonons $\bar{n}$ [Eq.(7)] at frequency $\omega_{\text {eff }}$ as a function of $T$. The vertical dotted line corresponds to the temperature $T_{c}$ at which thermal phonons of energy larger than $\hbar \omega_{\text {eff }}$ start exciting the rotor, degrading the entanglement. Parameters of Table 凹

We remark that the temperature in Fig. $2 T$ is not the temperature of the mirror; as is well known the presence of red-detuned laser light in the cavity can increase the mirror damping from $D_{\phi}$ to $D_{\text {eff }}$ and thus lower the effective temperature of the mirror $T_{\text {eff }}$ significantly below $T$. More precisely,

$$
T_{\text {eff }}=\left(\frac{D_{\phi}}{D_{\text {eff }}}\right) T .
$$

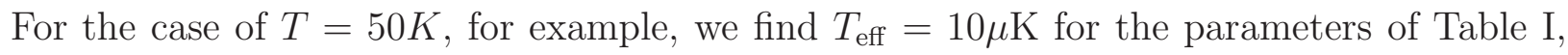
where $D_{\text {eff }}$ is (see Eq. (7) of Ref. [18]),

$$
\begin{aligned}
D_{\text {eff }} & =D_{\phi}+\frac{2 \xi_{\phi}^{2} \gamma P_{\text {in }}}{\omega_{c}}\left(\frac{\Delta}{\Delta^{2}+(\gamma / 2)^{2}}\right) \\
& \times \frac{\gamma}{\left[(\gamma / 2)^{2}+(\omega-\Delta)^{2}\right]\left[(\gamma / 2)^{2}+(\omega+\Delta)^{2}\right]}
\end{aligned}
$$

Here $\omega$ is the response frequency of the system, taken in our case to be $\omega_{\text {eff }}$ [see Eq.(29)].

\section{B. Low temperature regime}

Figure 3 is a blown-up view of the portion of Fig. 2 for low ambient temperatures. The entanglement is maximum at $T \sim 0$, as would be intuitively expected. It remains at that value as the temperature is increased until a temperature $T_{c}$ where it undergoes a sharp decrease. At that temperature the peak frequency of the thermal phonons becomes larger 


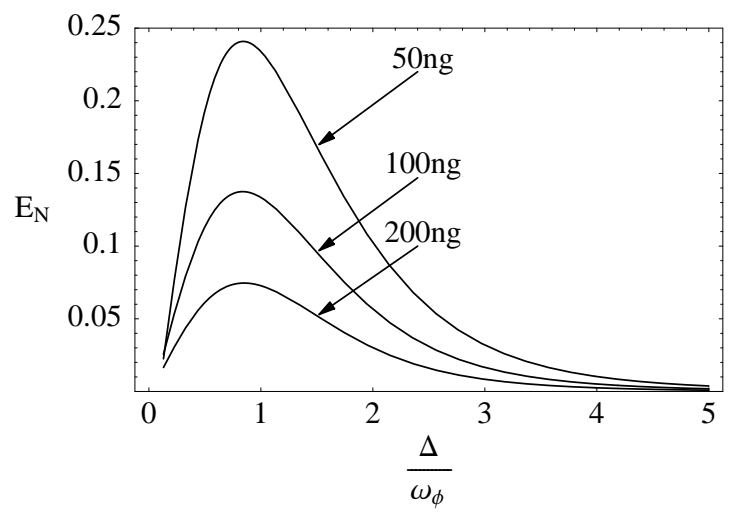

FIG. 4: The logarithmic negativity $E_{N}$ plotted as a function of the detuning $\Delta$ (normalized by the angular frequency $\omega_{\phi}$ of the rotating mirror) of the laser radiation from the cavity resonance. The three different curves correspond to different masses of the rotating mirror. The maximum of the entanglement occurs practically at $\Delta=\omega_{\text {eff }} \sim 0.8 \omega_{\phi}$ (see the discussion in Section IVC) for all three curves. For this plot we used the parameters from Table \except for the following changes : $L=0.1 \mathrm{~mm}, R=250 \mu \mathrm{m}, Q_{\phi}=10^{7}, F=10^{4}$, and $T=400 \mathrm{mK}$.

than $\omega_{\text {eff }}$ and they are capable of randomly exciting the rotating mirror, thus degrading its entanglement with the cavity mode.

This point is further illustrated in Fig. 3, which shows the mean number of thermal phonons $\bar{n}$ at frequency $\omega_{\text {eff }}$, [Eq.(77)] as a function of $T$. It increases noticeably for temperatures above $T_{c}$ and is clearly anti-correlated to $E_{N}$. This is consistent with an approximate calculation that shows that to lowest order in $\bar{n}$ we have

$$
E_{N} \simeq E_{0}-\kappa \bar{n}
$$

where $E_{0}$ is the maximum value of the entanglement and $\kappa$ is a constant whose value is a function of the parameters of the system. The analytical forms of $E_{0}$ and $\kappa$ are involved. For the parameters of Table \ their values are $E_{0} \sim 0.5$ and $\kappa \sim 3.1 \times 10^{-6}$.

\section{Detuning}

Fig. 4 shows the logarithmic negativity as a function of the detuning $\Delta=\omega_{c}-\omega_{L}-\phi_{s}=$ $\omega_{c}-\omega_{L}-g^{2}\left|a_{s}\right|^{2} / \omega_{\phi}$ for three masses of the rotating mirror. This result may be compared to Fig. 1 of Ref.[9]. A careful examination of the detuning results reveals that for each mass the entanglement is maximized when the detuning is equal to the mirror's effective angular 
frequency of rotation $\Delta=\omega_{\text {eff }}$, As is known, the presence of red-detuned radiation $(\Delta>0)$ causes anti-trapping and thus lowers the effective mechanical frequency of the mirror [5]. The effective frequency $\omega_{\text {eff }}$ is given explicitly by

$$
\begin{aligned}
\omega_{\mathrm{eff}}^{2} & =\omega_{\phi}^{2}-\frac{2 \xi_{\phi}^{2} \gamma P_{\text {in }}}{I \omega_{c}}\left(\frac{\Delta}{\Delta^{2}+(\gamma / 2)^{2}}\right) \\
& \times \frac{\left((\gamma / 2)^{2}-\left(\omega^{2}-\Delta^{2}\right)\right.}{\left[(\gamma / 2)^{2}+(\omega-\Delta)^{2}\right]\left[(\gamma / 2)^{2}+(\omega+\Delta)^{2}\right]}
\end{aligned}
$$

hence $\omega_{\text {eff }}$ scales as $1 / \sqrt{I}$, that is, as the inverse square root of the mass. Note however that for the parameters of Fig. 4, $\omega_{\text {eff }} \sim 0.8 \omega_{\phi}$ for all choices of mass. Thus the maximum entanglement occurs for $\Delta / \omega_{\phi} \sim 0.8$.)

Physically, the condition $\Delta=\omega_{\text {eff }}$ corresponds to the optimum detuning for optomechanical cooling [30] and can be understood in terms of cavity enhanced scattering of the anti-Stokes photons by the rotating mirror [4]. Thus it is not surprising that $E_{N}$ reaches a maximum at this detuning.

\section{Angular momentum}

As pointed out in Ref. [18], in the case of optical cooling of a vibrating mirror the linear momentum of the photons is difficult to change experimentally, and essentially entails replacement of the laser radiation source. For example to increase the linear momentum just by a factor of two would require an ultraviolet laser in place of an infrared laser. In contrast in the case of rotational cooling it is comparatively easier to achieve higher $l$. In light of this fact we also studied the entanglement as a function of the orbital angular momentum carried away from the mirror by the Laguerre-Gaussian mode,. The results of this analysis are summarized in Fig. 5, For the temperature $T=10 \mathrm{~K}$ considered in the figure, entanglement appears only beyond the threshold value $l_{c} \sim 21$. This is consistent with Eq. (3) which shows that for sufficiently low angular momentum, i.e. $l<l_{c}$, the optorotational coupling between radiation and the rotating mirror may not be high enough

for entanglement to occur. The value of $l_{c}$ depends on the other parameters that contribute to the optorotational coupling and of course also on the ambient temperature $T$. In general we found that entanglement appears when the effects of radiation torque coupling $g$ are strong enough to counter the thermal noise, which increases perceptibly with temperature beyond $T=T_{c}$. 


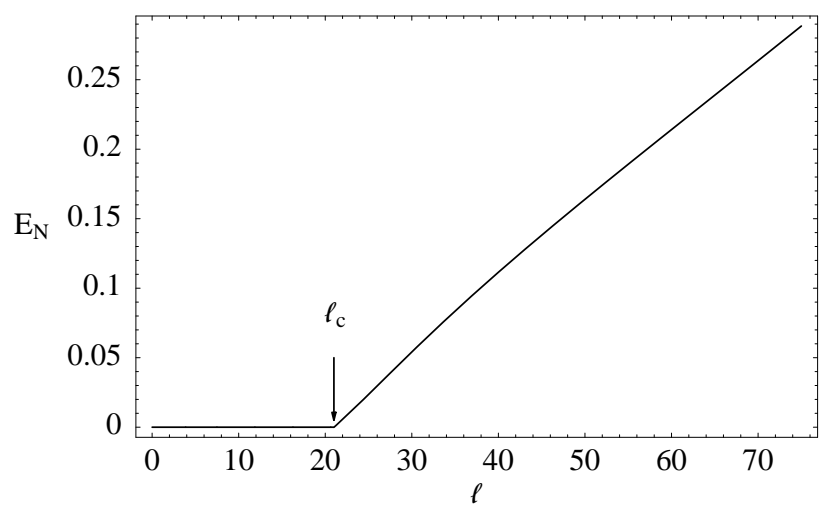

FIG. 5: Logarithmic negativity $E_{N}$ as a function of the orbital angular momentum carried by the Laguerre-Gaussian mode. The threshold angular momentum $l_{c} \sim 21$ at which entanglement begins

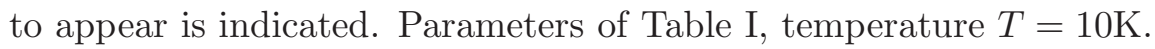

\begin{tabular}{|c|c|c|c|c|}
\hline No. & Parameter & Description & Value & Units \\
\hline \hline 1. & $L$ & Cavity length & 1 & $\mathrm{~mm}$ \\
\hline 2. & $\lambda$ & Laser wavelength & 810 & $\mathrm{~nm}$ \\
\hline 3. & $\omega_{c}$ & Cavity resonance frequency & $2 \pi 10^{14}$ & $\mathrm{~Hz}$ \\
\hline 4. & $\omega_{\phi}$ & Rotating mirror angular frequency & $2 \pi 10$ & $\mathrm{MHz}$ \\
\hline 5. & $M$ & Rotating mirror mass & 100 & $\mathrm{ng}$ \\
\hline 5. & $R$ & Rotating mirror radius & 10 & $\mu \mathrm{m}$ \\
\hline 7. & $Q_{\phi}$ & Mechanical quality factor & $2 \times 10^{6}$ & - \\
\hline 8. & $F$ & Optical finesse & $5 \times 10^{3}$ & - \\
\hline 9. & $l$ & Orbital angular momentum & 100 & $\hbar$ \\
\hline 10. & $P_{\text {in }}$ & Input laser power & 50 & $\mathrm{~mW}$ \\
\hline 11. & $\Delta / \omega_{\phi}$ & Normalized laser detuning & 1 & - \\
\hline \hline
\end{tabular}

TABLE I: Definitions and approximate values of some of the parameters used in the text. 


\section{CONCLUSION}

To conclude, we have demonstrated theoretically that radiation torque can serve to entangle a Laguerre-Gaussian cavity field with a rotating spiral phase element. We have shown that the physical basis of entanglement is the exchange of angular momentum between the cavity mode and the rotating mirror. The mirror-field entanglement was investigated as a function of temperature and shown to be measurable at the temperatures routinely achieved by cryogenic apparatus.

The entanglement was also shown to remain at its maximum value until a critical temperature at which the peak of the thermal spectrum occurred at the frequency corresponding to the rotor quanta, and beyond which the random excitation due to the thermal photons degraded the entanglement. The behavior of the entanglement with respect to cavity detuning was shown to be consistent with the mechanism of optomechanical cooling, i.e. the entanglement was maximum at a detuning known to correspond to optimal cooling of the mirror. Further, the fact that the angular momentum of the light can be changed relatively easily justified examining the entanglement with respect to changes in angular momentum. The main result here is that the mirror-radiation coupling only becomes strong enough to overcome the thermal noise and yield entanglement beyond a critical value of the angular momentum.

The measurement of the entanglement investigated in this paper can be carried out in a manner analogous to that proposed in the case of a linearly vibrating cavity and illustrated in Fig. 3 of Ref. [9]. In the present case the mirrors are replaced by spiral phase elements, and a second optical cavity can be formed by placing a third transmissive spiral phase element beyond the rotating mirror of Fig. 1. By using a weak intracavity field for this second cavity all the elements of the correlation matrix $C$ can be obtained as proposed in Ref. [9] and demonstrated in a measurement of the entanglement between two optical fields [25].

\section{Acknowledgments}

This work is supported in part by the US Office of Naval Research, by the National Science Foundation and by the US Army Research Office. We acknowledge fruitful discussions with H. Uys, O. Dutta and M. Leclerc. 


\section{APPENDIX A: LYAPUNOV EQUATION}

Here we derive Eq.(24), which is a typical Lyapunov equation encountered in the study of dynamical systems. Also, we show our method for solving the equation.

\section{Derivation}

Beginning from Eq.(23)

$$
C=\int_{0}^{\infty} d s M(s) D M(s)^{T}
$$

where $M(s)=e^{B s}$ and the matrices $B, D$ are given by Eqs.(13) and (22) respectively, we integrate by parts using $f^{\prime}(s)=e^{B s}$ and $g(s)=D e^{B^{T} s}$ in the rule

$$
\int_{0}^{\infty} d s f^{\prime}(s) g(s)=[f(s) g(s)]_{0}^{\infty}-\int_{0}^{\infty} d s f(s) g^{\prime}(s) .
$$

This yields the equation

$$
\begin{aligned}
C & =\left[B^{-1} e^{B s} D e^{B^{T} s}\right]_{0}^{\infty}-\int_{0}^{\infty} d s B^{-1} e^{B s} D e^{B^{T} s} B^{T} \\
& =-B^{-1} D-B^{-1}\left(\int_{0}^{\infty} d s e^{B s} D e^{B^{T} s}\right) B^{T} \\
& =-B^{-1} D-B^{-1} C B^{T}
\end{aligned}
$$

where in the second step we have used the fact if the Routh-Hurwitz criterion is satisfied then $\lim _{s \rightarrow \infty} e^{B s}=0$, since all the eigenvalues of $B$ have negative real parts. We then pre-multiply the last line of Eq. (A3) by $B$ and rearrange to obtain

$$
B C+C B^{T}=-D
$$

which is Eq. (24).

\section{Solution}

Here we record our method of solving Eq. (A4) for the correlation matrix $C$. By definition [see above Eq. (21)] $C$ is a $4 \times 4$ real symmetric matrix. Thus we can write

$$
C=\left(\begin{array}{cccc}
\lambda_{1} & a_{1} & a_{2} & a_{3} \\
a_{1} & \lambda_{2} & b_{1} & b_{2} \\
a_{2} & b_{1} & \lambda_{3} & c_{1} \\
a_{3} & b_{2} & c_{1} & \lambda_{4}
\end{array}\right)
$$


where we have denoted the diagonal elements with Greek letters and the off-diagonal with Roman letters. We now define a matrix $C^{\prime}$ where

$$
C^{\prime}=B C+C B^{T}+D
$$

which equals zero as per Eq. (24). Evaluating the RHS of Eq. (A6) we find that at least one matrix element of $C^{\prime}$ involves a single non-diagonal element of $C$. This observation, which is central to our method, turns out to be true at every iteration, where the process of iteration is defined below.

We now solve for the non-diagonal element of $C$ mentioned above by equating the relevant matrix element of $C^{\prime}$ to zero. We then use that value of the non-diagonal element in $C$ and re-evaluate $C^{\prime}$. This process is performed iteratively until all the off-diagonal elements of $C$ are expressed in terms of the diagonal elements of $C$. At this stage $C$ has the form

$$
C=\left(\begin{array}{llll}
\lambda_{1} & 0 & \frac{\left(\lambda_{1}-\lambda_{2}\right) \omega_{\phi}}{G} & \frac{\gamma\left(\lambda_{3}+\lambda_{4}-1\right)}{2 G} \\
0 & \lambda_{2} & -\frac{\gamma_{\phi}\left(2 \bar{n}-2 \lambda_{2}+1\right)}{2 G} & \frac{\gamma^{2}\left(\lambda_{3}+\lambda_{4}-1\right)-4 \Delta \lambda_{2} \omega_{\phi}-4 \lambda_{1}\left(G^{2}-\Delta \omega_{\phi}\right)}{4 G \omega_{\phi}} \\
\frac{\left(\lambda_{1}-\lambda_{2}\right) \omega_{\phi}}{G} & -\frac{\gamma_{\phi}\left(2 \bar{n}-2 \lambda_{2}+1\right)}{2 G} & \lambda_{3} & \frac{\gamma\left(2 \lambda_{3}-1\right)}{4 \Delta} \\
\frac{\gamma\left(\lambda_{3}+\lambda_{4}-1\right)}{2 G} & \frac{\gamma^{2}\left(\lambda_{3}+\lambda_{4}-1\right)-4 \Delta \lambda_{2} \omega_{\phi}-4 \lambda_{1}\left(G^{2}-\Delta \omega_{\phi}\right)}{4 G \omega_{\phi}} & \frac{\gamma\left(2 \lambda_{3}-1\right)}{4 \Delta} & \lambda_{4}
\end{array}\right)
$$

Also we find $C^{\prime}=0$ yields 4 equations in the diagonal elements $\lambda_{1,2,3,4}$ of $C$, and these can be easily solved, giving us analytical expressions for all the elements of the $C$ matrix.

[1] S. Gigan, H. R. Böhm, M. Paternostro, F. Blaser, G. Langer, J. B. Hertzberg, K. C. Schwab, D. Bäuerle, M. Aspelmeyer, and A. Zeilinger, Nature 444, 67 (2006).

[2] D. Kleckner and D. Bouwmeester, Nature 444, 75 (2006).

[3] O. Arcizet, P. -F. Cohadon, T. Briant, M. Pinard, and A. Heidmann, Nature 444, 71 (2006).

[4] A. Schliesser, P. Del'Haye, N. Nooshi, K. J. Vahala, and T. J. Kippenberg, Phys. Rev. Lett. 97, 243905 (2006).

[5] T. Corbitt, Y. Chen, E. Innerhofer, H. Muller-Ebhardt, D. Ottaway, H. Rehbein, D. Sigg, S. Whitcomb, C. Wipf, and N. Mavalvala, Phys. Rev. Lett 98, 150802 (2007).

[6] J. D. Thompson, B. M. Zwickl, A. M. Jayich, F. Marquardt, S. M. Girvin, and J. G. E. Harris, arXiv:0707.1724v2[quant-ph](2007). 
[7] M. A. Nielsen and I. L. Chuang, Quantum Computation and Quantum Information (Cambridge University Press, Cambridge, 2000), 1st ed.

[8] S. Pirandola, S. Mancini, D. Vitali and P. Tombesi, Phys. Rev. A 68, 062317 (2003).

[9] D. Vitali, S. Gigan, A. Ferreira, H. R. Bohm, P. Tombesi, A. Guerreiro, V. Vedral, A. Zeilinger and M. Aspelmeyer, Phys. Rev. Lett. 98, 030405 (2007).

[10] S. Mancini, V. Giovannetti, D. Vitali and P. Tombesi, Phys. Rev. Lett. 88, 120401 (2002).

[11] M. Pinard, A. Dantan, D. Vitali, O. Arcizet, T. Briant and A. Heidmann, Europhys. Lett. $72,747(2005)$.

[12] S. Pirandola, D. Vitali, P. Tombesi and S. Lloyd, Phys. Rev. Lett. 97, 150403 (2006).

[13] J. Zhang, K. Peng and S. L. Braunstein, Phys. Rev. A 68, 013808 (2003).

[14] V. Giovannetti, S. Mancini and P. Tombesi, Europhys. Lett. 54, 559 (2001).

[15] S. Bose, K. Jacobs and P. L. Knight, Phys. Rev. A 56, 4175 (1997).

[16] S. Mancini and A. Gatti, J. Opt.B: Quantum Semiclasss. Opt. 3, S66 (2001).

[17] S. Mancini, D. Vitali and P. Tombesi, Phys. Rev. Lett. 90, 137901 (2003).

[18] M. Bhattacharya and P. Meystre, Phys. Rev. Lett. (in press), arXiv:0705.0784.

[19] S. S. R. Oemrawsingh, E. R. Eliel, J. P. Woerdman E. J. K. Verstegen, J. G. Kloosterboer and G. W. 't Hooft, J. Opt. A : Pure Appl. Opt. 6, S288 (2004).

[20] C. Gardiner, Quantum Noise (Springer-Verlag, Berlin, 1991).

[21] P. Meystre, E. M. Wright, J. D. McCullen, and E. Vignes, J. Opt. Soc. Am. B 2, 1830 (1985);

J. D. McCullen, P. Meystre, and E. M. Wright, Optics Letters 9, 193 (1984).

[22] S. Mancini and P. Tombesi, Phys. Rev. A 49, 4055 (1994).

[23] E. X. DeJesus and C. Kaufman, Phys. Rev. A 35, 5288 (1987).

[24] G. Vidal and R. F. Werner, Phys. Rev. A 65, 032314 (2002).

[25] J. Laurat, G. Keller, J. A. Oliveira-Huguenin, C. Fabre, T. Coudreau, A. Serafini, G. Adesso and F. Illuminati, J. Opt. B : Quantum Semiclasss. Opt. 7, S577 (2005).

[26] A. Serafini, F. Illuminati, M. G. A. Paris and S. DeSiena, Phys. Rev. A 69, 022318 (2004).

[27] G. Adesso, A. Serafini and F. Illuminati, Phys. Rev. A 70, 022318 (2004).

[28] M. Poggio, C. L. Degen, H. J. Mamin, and D. Rugar, Phys. Rev. Lett. 99, 017201 (2007).

[29] We have repeated the calculation of Ref. [9] and found the same behavior.

[30] F. Marquardt, J. P. Chen, A. A. Clerk and S. M. Girvin, Phys. Rev. Lett. 99, 093902 (2007). 